\title{
Aproveitamento de resíduos madeireiros de Pinus sp. com diferentes granulometrias para a produção de briquetes
}

\section{Use of wood residues of Pinus sp. with different granulometry to briquettes production}

\author{
Lucas Henrique Oliveira ${ }^{1}$, Pedro Vilela Gondim Barbosaํ․, Pedro Augusto Fonseca Lima ${ }^{1}$, \\ Fábio Minoru Yamaji² e Carlos Roberto Sette Júnior ${ }^{1, *}$
}

${ }_{1}^{1}$ Departamento de Engenharia Florestal. UFG - Universidade Federal de Goiás. Rodovia Goiânia - Nova Veneza, km O - Campus Samambaia - Caixa Postal 131 - 74690-900 - Goiânia, GO, Brasil 2Departamento de Ciências Ambientais. UFSCAR - Universidade Federal de São Carlos. Rodovia João Leme dos Santos km 110 - 18052-780 - Sorocaba, SP, Brasil

( ${ }^{\star}$-mail: crsettejr@hotmail.com)

http://dx.doi.org/10.19084/RCA17010

Recebido/received: 2017.01.18

Recebido em versão revista/received in revised form: 2017.03.15

Aceite/accepted: 2017.04.05

\begin{abstract}
R E S U M O
O presente trabalho teve como objetivo avaliar o efeito da granulometria da biomassa residual da madeira de Pinus sp. na qualidade de briquetes. A biomassa residual utilizada foi obtida do processo industrial de processamento da madeira de Pinus sp., sendo classificada em (i) serragem; material residual do processo industrial apresentando granulometria diversa e (ii) moída; material moído e classificado na peneira de 60 mesh. Da biomassa foram determinados os teores de materiais voláteis, cinzas e carbono fixo, poder calorífico superior, densidade a granel e energética e produzidos briquetes em briquetadeira laboratorial com temperatura de $120^{\circ} \mathrm{C}$, tempo de prensagem de 5 minutos, pressão de $140 \mathrm{kgf.cm}{ }^{-2}$ e tempo de resfriamento de 10 minutos. A qualidade dos briquetes foi determinada pelas variáveis energéticas e físico-mecânicas: densidade aparente e energética, durabilidade, resistência à tração por compressão diametral e expansão volumétrica. Os resíduos de biomassa de Pinus sp. provenientes do processamento da madeira são adequados para a confecção de briquetes. Não houve efeito significativo da granulometria da biomassa de Pinus sp. nas características energéticas e físico-mecânicas dos briquetes. A utilização da biomassa residual obtida diretamente do processamento da madeira de Pinus sp. é recomendada para produção de briquetes em detrimento a biomassa mais fina, com 60 mesh, pois a ela estão atrelados menores custos de produção, sem a necessidade de reprocessamento da matéria-prima e adequação granulométrica.
\end{abstract}

Palavras-chave: serragem, briquetagem, bioenergia.

\begin{abstract}
A B S T R A C T
The present work aims to evaluate the effect of the biomass granulometry of Pinus sp. on the quality of briquettes. The residual biomass used was obtained from the industrial processing of Pinus sp. wood, being classified in (i) sawdust; residual material of the industrial process presenting diverse granulometry and (ii) ground; material graded and classified in 60 mesh sieve. The proximate analysis (volatile, ash and fixed carbon contents), highest calorific value, bulk and energy density of the biomass was determined and produced briquettes with temperature of $120^{\circ} \mathrm{C}$, pressing time of 5 minutes at a pressure of $140 \mathrm{kgf.cm}^{-2}$ and cooling time of 10 minutes. The quality of briquettes was determined by the energy and physical-mechanical variables: bulk and energetic density, durability, tensile strength by diametrical compression and expansion. The residues of Pinus sp. are suitable for the production of briquettes. There was no effect of biomass granulometry in the energetic and physico-mechanical characteristics of briquettes. The use of residual biomass obtained directly from the processing of Pinus sp. is recommended for the production of briquettes in detriment to the finer biomass with 60 mesh, because it is linked to lower production costs.
\end{abstract}

Keywords: sawdust, briquetting, bioenergy. 


\section{INTRODUÇÃO}

Atualmente no Brasil, 41,2\% da matriz energética é baseada em fontes renováveis, sendo $8,2 \%$ de biomassa florestal e agrícola (Brasil, 2016). No entanto, o país tem potencial para aumentar a participação da biomassa na matriz energética, entre outros fatores, devido a grande quantidade de resíduos gerados nos setores agrícola e florestal. A diversificação da matriz energética e novas soluções para o uso racional e eficiente dos recursos naturais são estratégias que podem aumentar a oferta de energia e atender a demanda do Brasil.

A biomassa florestal, para geração de energia, pode ser obtida na colheita, em tratos silviculturais, no processamento da madeira ou de florestas energéticas, que tem como finalidade o fornecimento de matéria prima para energia (Spanhol et al., 2015).

A indústria de processamento da madeira brasileira apresenta, em média, um aproveitamento baixo, em torno de $40 \%$, sendo o restante transformado em resíduos (Brand, 2010). Estima-se que no Brasil a produção de resíduos florestais esteja na ordem de 17 milhões de metros cúbicos por ano (FAO, 2015) e com os crescentes aumentos nos custos dos insumos energéticos, as indústrias têm procurado o aproveitamento dos resíduos industriais e florestais como fonte alternativa de energia (Chen et al., 2009).

Os principais problemas da utilização de resíduos industriais e florestais para produção de energia estão relacionados, principalmente, a baixa densidade energética, as dimensões e volumes variados, a alta higroscopicidade e teor de umidade (Santos et al., 2013). Uma das formas de solucionar os problemas e melhorar as propriedades dos resíduos é a densificação da biomassa, ou seja, produção de materiais sólidos de tamanho médio com alta concentração de energia denominados briquetes ou pellets (Carvalho et al., 2013).

Nesse contexto, os briquetes foram criados em função da demanda por um novo tipo de combustível compactado com alta densidade energética, para o transporte a maiores distâncias, otimização do armazenamento e principalmente para o uso em equipamentos de queima mais modernos (Protásio et al., 2015). As características e qualidade dos briquetes podem ser influenciados por variáveis relacionadas à matéria prima (granulometria, composição química da biomassa, teor de umidade etc.) e ao processo de produção (pressão, temperatura etc.) (Furtado et al., 2010), sendo fundamental o estudo dos efeitos destas variáveis na qualidade dos briquetes, visando apresentar soluções no atendimento da demanda energética, principalmente pelo aproveitamento da matéria-prima residual de processos industriais, como na produção de briquetes, fundamentais para uma destinação viável, econômica e ambientalmente correta desses resíduos.

Alguns trabalhos científicos indicam na literatura especializada a viabilidade técnica e econômica do aproveitamento dos resíduos madeireiros (Farage et al., 2013; Gonçalves et al., 2013; Protásio et al., 2015) como fonte de energia e indicam a necessidade do desenvolvimento de tecnologias e processos que permitam o reaproveitamento e disposição final adequada destes resíduos, como é o caso da briquetagem. Apesar dos trabalhos indicados, são escassos no Brasil, estudos relacionados a utilização dos resíduos do processamento da madeira de Pinus sp. e que visem agregar valor energético a biomassa, uma vez que grande parte do resíduo produzido é aplicado na queima direta em fornos e caldeiras ou como forração de cama de frango em granjas.

O presente trabalho teve como objetivo determinar a viabilidade técnica do aproveitamento da biomassa residual de Pinus sp., com diferentes granulometrias, para produção de briquete.

\section{MATERIAIS E MÉTODOS}

\section{Coleta e preparo do material}

A biomassa residual utilizada para determinar o efeito da granulometria na qualidade de briquetes foi obtida do processamento industrial da madeira de Pinus sp. A biomassa foi coletada diretamente da serraria e transportada até o Laboratório de Qualidade da Madeira e Bioenergia (LQMBIO) do Departamento de Engenharia Florestal da Universidade Federal de Goiás (UFG), Goiânia, GO, Brasil, para realizações das análises. 
Inicialmente, o material foi classificado granulometricamente, sendo submetido a uma separação mecânica utilizando peneiras classificadas em 20, 40, 60 e 100 mesh com auxílio de um agitador de peneiras com batidas intermitentes. Para a avaliação do efeito do tamanho das partículas (granulometria) na qualidade dos briquetes, foram definidas duas condições: (i) material original, obtido diretamente do processo industrial, chamado de serragem e que apresenta como característica partículas com diferentes granulometrias e (ii) material constituído apenas de partículas com 60 mesh, chamado de moído.

\section{Caracterização da biomassa}

A caracterização química da biomassa do resíduo de Pinus sp. foi realizada no material a 60 mesh, conforme preconizado pela Associação Brasileira de Normas Técnicas (ABNT - NBR). Foram determinados os teores de cinza, materiais voláteis e teor de carbono fixo de acordo com a NBR 8112/1986 (ABNT, 1986).

A densidade a granel foi determinada para a biomassa obtida diretamente do processo industrial, de acordo com a NBR 6922/1981 (ABNT, 1981): relação entre a massa de biomassa e o volume conhecido de um recipiente.

O valor do poder calorífico superior (PCS) foi obtido experimentalmente através da bomba calorimétrica, por meio da metodologia estabelecida na norma NBR 8633/1984 (ABNT, 1984) e calculada a densidade energética da biomassa, a partir do produto entre o valor do PCSe da densidade a granel.

\section{Produção e análise dos briquetes}

O teor de umidade da biomassa residual nas duas condições (serragem e moída) foi ajustado para $12 \%$ para posterior briquetagem em briquetadeira laboratorial, com temperatura de $120^{\circ} \mathrm{C}$, pressão $140 \mathrm{kgf.cm}{ }^{-2}$, tempo de compactação de 5 minutos e resfriamento de 10 minutos.

O teor de umidade de $12 \%$ foi escolhido por estar dentro da faixa considerada ideal para a fabricação de briquetes (Konishi et al., 2011) e as condições de briquetagem foram definidas experimentalmente a partir de testes preliminares de tempo de prensagem e de resfriamento, sendo escolhidos aqueles em que os briquetes apresentaram as melhores formações, conforme proposto por Vilas Boas (2011). A pressão exercida está dentro da faixa utilizada por diversos trabalhos (Quirino et al., 2012) e a temperatura teve como objetivo a plasticização da lignina (Chen et al., 2009), atuando como ligante natural das partículas durante a compactação.

Para cada briquete utilizou-se $40 \mathrm{~g}$ de biomassa, obtendo-se ao final um briquete de aproximadamente $4 \mathrm{~cm}$ de comprimento e $3 \mathrm{~cm}$ de diâmetro, tendo sido produzidos 20 briquetes por condição (serragem e moída), totalizando 40 briquetes.

A densidade aparente de 5 briquetes por condição, foi determinada por meio do método estequiométrico, o qual consiste em obter o volume a partir de medições, utilizando um paquímetro e a massa do briquete em uma balança com precisão de 0,001 gramas. Foi obtida através da equação: dap $=\mathrm{M}_{\mathrm{i}} / \mathrm{V}$, onde: dap $=$ Densidade aparente $\left(\mathrm{g} \cdot \mathrm{cm}^{-3}\right)$; $\mathrm{M}_{\mathrm{i}}=$ Massa inicial (g); $\mathrm{V}=$ Volume individual $\left(\mathrm{cm}^{3}\right)$.

A resistência a tração por compressão diametral foi realizada em 5 briquetes por condição empregando-se uma máquina universal de ensaios EMIC DL30000, com célula de carga de 500 kgf, a uma velocidade constante de $0,3 \mathrm{~mm} \cdot \mathrm{min}^{-1}$ (Protássio et al., 2011; Quirino et al., 2012; Souza, 2012), onde uma carga em sentido transversal é aplicada sobre as amostras. O ensaio foi realizado a partir de uma adaptação da norma NBR 7222/1994 (ABNT, 1994) para determinação da resistência a tração por compressão diametral em amostras cilíndricas de concreto e argamassa.

A durabilidade foi determinada por perda de massa das amostras, conforme descrito por Toscano et al. (2013) e Liu et al. (2014). Cinco briquetes por condição foram pesados para a obtenção da massa inicial e na sequência colocados em uma peneira com tela de 2,83 $\mathrm{mm}$ de tamanho, posteriormente foram levados a um agitador de peneiras, permanecendo por 10 minutos, a 80 rotações por minuto. Após este procedimento, os briquetes foram novamente pesados e obtidos a massa final. A durabilidade foi calculada a partir da equação: Dur=100 $\left[\mathrm{M}_{\mathrm{id}} \mathrm{M}_{\mathrm{fd}} / \mathrm{M}_{\mathrm{id}}\right] \times 100$, onde: Dur: Durabilidade 
do briquete (\%); $\mathrm{M}_{\mathrm{id}}$ : Massa inicial da amostra (g); $\mathrm{M}_{\mathrm{fd}}$ : Massa final da amostra $(\mathrm{g})$.

A expansão volumétrica dos briquetes foi calculada pela mensuração da altura e do diâmetro de 5 briquetes por condição e realizado o cálculo do volume em dois momentos diferentes: (i) imediatamente após a briquetagem e (ii) 72 horas após a briquetagem - intervalo de tempo necessário para a estabilização dimensional dos briquetes. A expansão foi calculada pela equação: $\operatorname{Exp}=\left[\left(\mathrm{Vol}_{2} \mathrm{Vol}_{1}\right) / \mathrm{Vol}_{1}\right] \times 100$, onde: Exp: expansão volumétrica dos briquetes (\%); $\mathrm{Vol}_{1}$ : volume do briquete imediatamente após a compactação $\left(\mathrm{cm}^{3}\right)$; $\mathrm{Vol}_{2}$ : volume do briquete transcorrido certo tempo após a compactação $\left(\mathrm{cm}^{3}\right)$.

\section{Análise estatística}

$\mathrm{Na}$ análise estatística dos resultados foi utilizado o delineamento inteiramente casualizado, sendo aferidos os "outliers", distribuição dos dados e heterogeneidade da variância. Para as características da biomassa, foram obtidas as médias e calculado do desvio padrão. Nos resultados da densidade aparente, energética, durabilidade, resistência a tração por compressão diametral e expansão volumétrica dos briquetes aplicou-se a análise de variância (ANOVA) para verificar o efeito da condição do material (serragem e moída), a 5\% de probabilidade.

\section{RESULTADOS E DISCUSSÃO}

\section{Caracterização da biomassa}

A caracterização da biomassa obtida diretamente do processo industrial de processamento da madeira de Pinus sp. iniciou-se pela sua classificação granulométrica, tendo sido observadas partículas com diferentes tamanhos (Figura 1): a maior parte das partículas foram classificadas em 20 mesh, (34\%), seguido das de 40 e 60 mesh, com 27 e $28 \%$, respectivamente. A menor parte da biomassa residual foi caracterizada como retida na peneira de 100 mesh, com $11 \%$. Apesar da heterogeneidade das partículas da biomassa residual, todo o material utilizado é considerado na nomenclatura como "fino"(Bergström et al., 2008; Quirino et al., 2012) pois se encontra abaixo de $1 \mathrm{~mm}$.

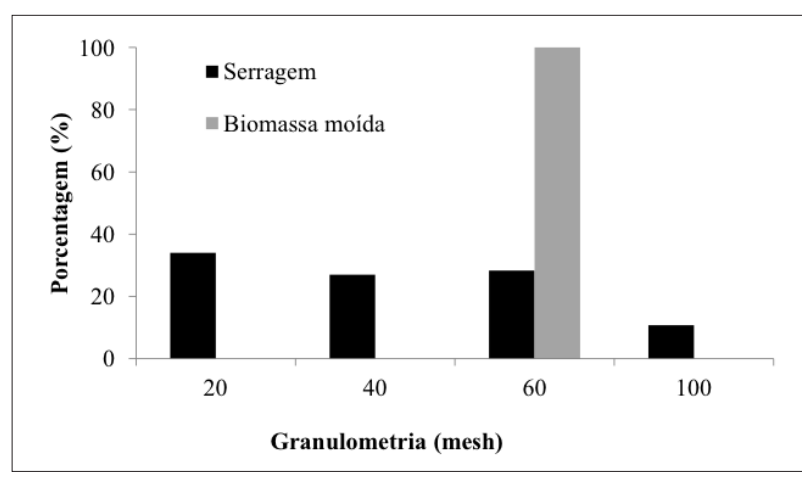

Figura 1 - Porcentagem granulométrica das biomassas utilizadas para produção dos briquetes.

Com relação aos materiais voláteis, a biomassa de Pinus sp. apresentou teor médio de 82\% (Figura 2). A quantidade de materiais voláteis está diretamente relacionada com a queima no processo de carbonização, pois quanto maior o teor de materiais voláteis, mais rápido é a queima. Amorim et al. (2015) avaliando diferentes tipos de biomassa de espécies florestais obteve valores médios de materiais voláteis de 85,3\% para Pinus sp., estando próximo ao obtido nesse estudo.

Quanto ao teor de cinzas, a biomassa apresentou um valor médio de $0,83 \%$ (Figura 2). Segundo Carroll e Finnan (2012), o valor médio do teor de cinzas encontrado para espécies de Pinus sp. é de 0,7\% e vários estudos demonstraram que a madeira de Pinus sp. atende as normas europeias e norte-americanas para produção de materiais sólidos, que exigem teores de cinza entre 0,5-1,5\% (Carroll e Finnam, 2012; Protásio et al., 2015).

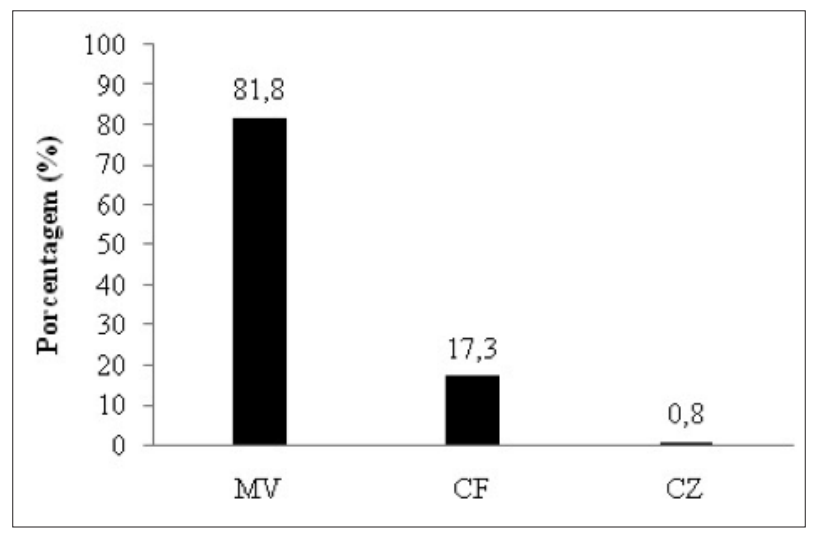

Figura 2 - Teores de materiais voláteis (MV), carbono fixo (CF) e cinzas (CZ) da biomassa de Pinus sp. 
Para o teor de carbono fixo, a biomassa obteve teor médio de $17,4 \%$, apresentando padrão de queima fundamental para produção de energia, pois Brito e Barrichello (1982), recomendam teores de carbono fixo entre 14 a $25 \%$ e afirmam que combustíveis com elevado índice de carbono fixo queimam mais lentamente, acarretando em elevado tempo de residência destes dentro dos queimadores. $\mathrm{O}$ valor encontrado foi próximo ao de Silva et al. (2015), ao avaliar a serragem de Pinus sp. para produção de briquetes, obtendo valor médio de $18,6 \%$.

A biomassa obtida diretamente do processo industrial de processamento da madeira de Pinus sp. apresentou densidade a granel média $149,8 \mathrm{~kg}$. $\mathrm{cm}^{3}$, com desvio padrão de 4,64 (Quadro 1), valor próximo ao observado por Silva et al. (2015), que constatou $180 \mathrm{~kg} . \mathrm{cm}^{3}$, para serragem de Pinus sp. A determinação da densidade a granel é de fundamental importância para gerar informações úteis para a logística e transporte da biomassa, uma vez que estima os espações vazios entre uma partícula e fornece dados reais dos volumes para o transporte.

Os valores de poder calorífico superior (Quadro 1) variaram entre 18,5 a $20,8 \mathrm{MJ} . \mathrm{kg}^{-1}$. Os resultados encontrados para essa variável estão próximos com os apresentados por Souza et al. (2012), onde o poder calorífico superior variou entre, 19 e 21 MJ.kg-1 para biomassa florestal da espécie Pinus taeda. O PCS é um dos principais parâmetros para expressar a capacidade de geração de energia em substituição aos combustíveis derivados do petróleo. Essa propriedade está relacionada diretamente com a quantidade de energia liberada pela madeira em sua combustão, sendo importante para conhecer a capacidade energética da biomassa (Santos et al., 2011).

A densidade energética está diretamente relacionada com o poder calorífico da biomassa e com a densidade a granel. Portanto, o valor da densidade a granel encontrado para biomassa influenciou na densidade energética do material, encontrando valor médio de $2961 \mathrm{MJ} . \mathrm{m}^{-3}$, considerado baixo quando comparado a outros tipos de materiais. Protásio et al. (2011) obteve valor médio de densidade energética em torno de $3100 \mathrm{MJ} \cdot \mathrm{m}^{-3}$ para a serragem de eucalipto, utilizando a mesma foram de cálculo. É desejável que os resíduos lignocelulósicos apresentem maiores valores de densidade a granel, pois fatores como o custo do transporte e a densidade energética são essenciais na viabilidade econômica das energias renováveis (Santos et al., 2013).

A densidade energética é um importante parâmetro para combustíveis sólidos, pois, avalia a quantidade de energia armazenada em um determinado volume de material.

\section{Características energéticas e físico-mecânicas dos briquetes}

A granulometria da biomassa de Pinus sp. não influenciou as características energéticas e físico-mecânicas dos briquetes (Quadro 2): não foram observadas diferenças estatísticas entre as duas condições granulométricas (serragem e moída) para todas as variáveis analisadas. Este resultado

Quadro 1 - Valores do Poder calorífico superior (PCS), densidade à granel (D. granel) e energética (DE) da biomassa de Pinus sp.

\begin{tabular}{cccc}
\hline $\begin{array}{c}\text { Repetição } \\
\left(\mathbf{n}^{\circ}\right)\end{array}$ & D. granel $\left(\mathbf{K g}^{\left.-m^{-3}\right)}\right.$ & $\begin{array}{c}\text { PCS } \\
\left(\mathbf{M J} \cdot \mathbf{k g}^{-1}\right)\end{array}$ & $\begin{array}{c}\text { DE } \\
\left(\mathbf{M J . m}^{-3}\right)\end{array}$ \\
\hline 1 & 144,2 & 18,5 & 2667,7 \\
2 & 146,8 & 19,8 & 2906,6 \\
3 & 156,2 & 19,0 & 2967,8 \\
4 & 152,0 & 20,7 & 3146,4 \\
5 & 149,8 & 20,8 & 3115,8 \\
\hline Desvio Padrão & 4,64 & 1,03 & 195,9 \\
\hline Média & 149,8 & 19,8 & 2960,9 \\
\hline
\end{tabular}


Quadro 2 - Densidade aparente (DA),energética (DE), durabilidade (Dur), resistência à compressão diametral (RTCD) e expansão (Ex) dos briquetes

\begin{tabular}{cccccc}
\hline Condição & $\begin{array}{c}\text { DA } \\
(\text { Kg.m }\end{array}$ & $\begin{array}{c}\text { DE } \\
\left(\mathbf{M J} \cdot \mathbf{m}^{-3}\right)\end{array}$ & $\begin{array}{c}\text { Dur } \\
\mathbf{( \% )}\end{array}$ & $\begin{array}{l}\text { RTCD } \\
\mathbf{( M p a )}\end{array}$ & $\begin{array}{c}\text { Ex } \\
\mathbf{( \% )}\end{array}$ \\
\hline Serragem & $1218,20 \mathrm{a}$ & $24120,36 \mathrm{a}$ & $99,25 \mathrm{a}$ & $3,51 \mathrm{a}$ & $2,16 \mathrm{a}$ \\
& $(6,70)$ & $(132,3)$ & $(0,53)$ & $(0,17)$ & $(0,96)$ \\
\hline Moído & $1218,46 \mathrm{a}$ & $24125,51 \mathrm{a}$ & $99,50 \mathrm{a}$ & $3,41 \mathrm{a}$ & $2,88 \mathrm{a}$ \\
& $(8,32)$ & $(121,8)$ & $(0,21)$ & $(0,10)$ & $(1,81)$ \\
\hline
\end{tabular}

Médias seguidas da mesma letra, na coluna, não diferem entre si a 5\% de significância pelo Teste F. Desvio padrão entre parênteses.

pode estar associado as características dos materiais nas duas condições (serragem e moído), em que as porcentagens granulométricas (Figura 1) indicam a presença de partículas classificadas como finas, conforme já discutido.

Os briquetes produzidos nas duas condições apresentaram densidade aparente de $1218 \mathrm{~kg} . \mathrm{m}^{-3}$. O valor encontrado é considerado alto, sendo essa uma característica desejável quando se trabalha com combustíveis sólidos para geração de energia na forma de calor. Os briquetes de ambas condições obtiveram valores de densidade dentro do encontrado por Quirino et al. (2012), que trabalharam com resíduos de madeira de Eucalyptus sp., com valores de densidade aparente entre 1180 e $1340 \mathrm{~kg} . \mathrm{m}^{-3}$.

Observa-se um aumento na ordem de 8 vezes da densidade após a briquetagem (densidade a granel $149,8 \mathrm{~kg} \cdot \mathrm{m}^{-3}$ e densidade aparente $1218 \mathrm{~kg} \cdot \mathrm{m}^{-3}$ ). A densificação da biomassa através da produção dos briquetes, promove um aumento na densidade da biomassa in natura; este incremento da densidade após a compactação, evidencia a importância dos processos de compactação da biomassa para o melhor aproveitamento de materiais lignocelulósicos para a produção de bioenergia, pois o aumento na densidade dos briquetes representa uma diminuição do volume das biomassas, proporcionando uma maior concentração de massa em um mesmo espaço (Silva et al., 2015).

Da mesma forma do observado para a densidade, após a compactação da biomassa, observou-se o aumento da densidade energética (Quadro 2), evidenciando a importância da briquetagem no aproveitamento de resíduos lignocelulósicos para geração de energia, conforme já mencionado. Esse aumento ocorreu em função do aumento da densidade da biomassa ocasionado pela compactação, uma vez que o valor de PCS se mantém constante.

A durabilidade não difere entre os briquetes produzidos nas duas condições granulométricas, assim como o resultado obtido por Bergström et al. (2008), o qual evidenciou valores de durabilidade iguais em seus tratamentos (partículas finas, médias e grossas), mostrando que a durabilidade não é influenciada pela distribuição do tamanho das partículas.

A durabilidade é medida pela resistência do pellet ou briquete quanto ao choque e/ou atrito e corresponde a postura do material quanto ao desgaste mecânico, provocando a geração de partículas finas ou poeiras ao longo do transporte, transbordo e armazenagem, as quais representam um inconveniente para o consumidor e uma ameaça para a saúde (Vinterbäck, 2004). As partículas e poeiras também podem desestabilizar a regulação dos sistemas automatizados de aquecimento, bloquear os sistemas de alimentação das caldeiras e, consequentemente, findar a alimentação automatizada de combustível, ocasionar técnicas de combustão não homogêneas, além de elevar os riscos de incêndio e explosão no decorrer do manuseio, armazenamento e transporte (Li e Liu, 2000; Lehtikangas, 2001).

De acordo com estudo feito por Bergström et al. (2008) diferentes porcentagens granulométricas não influenciaram na produção de materiais densificados de Pinus sylvestris L., apresentando características físicas e termoquímicas semelhantes entre os tratamentos de diferentes granulometrias. Apesar do indicado por Bergström et al. (2008) e do observado no presente estudo, diferentes 
granulometrias misturadas podem influenciar a qualidade do briquete. Um exemplo é a caracterização de briquetes feitos a partir de diferentes granulometrias com resíduo de eucalipto realizado por Gonçalves et al. (2013), que demonstram os melhores valores de expansão e resistência a compressão para o briquetes produzido com maior amplitude granulométrica (10 a 100 mesh).

Apesar de não haver diferenças estatísticas nos valores de expansão (Ex) e resistência a tração por compressão diametral (RTCD), os briquetes produzidos a partir da serragem de Pinus sp. demonstraram tendência a características qualitativas superiores, ou seja, menor expansão e maior resistência (Quadro 2). Essa mesma tendência foi observada por Gonçalves et al. (2013) que obtiveram valores de expansão menores para materiais heterogêneos de maior amplitude granulométrica (10 - 100 mesh). Isso pode ser explicado, pois os espaços entre as partículas grandes, previamente ocupados por ar, passam a ser preenchidos com as partículas de tamanho menor, ocasionando maior coesão entre elas e consequentemente menor taxa de expansão. Vale ressaltar que os valores de desvio padrão para porcentagem de expansão são maiores que para as demais variáveis estudadas, pois ocorreu uma maior variação de valores entre as repetições da sua análise.
Foi observado por Gonçalves et al. (2013), melhores resultados de tensão suportada pelos corpos de prova para matéria-prima de serragem de Pinus sp., uma vez que partículas de diferentes tamanhos garantem uma grande superfície específica, gerando maior adesão entre as partículas. Essa adesão promove uma melhor compactação do material e, portanto, melhores resultados para resistência.

\section{CONCLUSÕES}

- Os resíduos de biomassa de Pinus sp. provenientes do processamento da madeira são adequados para a confecção de briquetes;

- Nãohouveefeitosignificativodagranulometriada biomassa dos resíduos de Pinus sp. nas características energéticas e físico-mecânicas dos briquetes;

A utilização da biomassa residual obtida diretamente do processo de processamento da madeira de Pinus sp. é recomendada para produção de briquetes em detrimento a biomassa mais fina, com 60 mesh, pois a ela estão atrelados menores custos de produção de briquetes, sem a necessidade de reprocessamento da matéria-prima e adequação granulométrica.

\section{REFERÊNCIAS BIBLIOGRÁFICAS}

Amorim, F.S.; Ribeiro, M.X.; Protásio, T.P.; Borges, C.H.A. \& Costa, R.M.C. (2015) - Produção de briquetes a partir de espécies florestais. Revista Verde de Agroecologia e Desenvolvimento Sustentável, vol. 10, n. 4, p. 34-41. http://dx.doi.org/10.18378/rvads.v10i4.3779

ABNT (1981) - Carvão vegetal: Ensaios físicos - Determinação da massa específica - Densidade a granel. Associação Brasileira de Normas Técnicas - ABNT, NBR 6922.

ABNT (1986) - Carvão vegetal: Análise Imediata. Associação Brasileira de Normas Técnicas - ABNT, NBR 8112. ABNT (1984) - Carvão vegetal: determinação do poder calorífico. Associação Brasileira de Normas Técnicas ABNT, NBR 8633.

ABNT (1994) - Argamassa e concreto: determinação da resistência à tração por compressão diametral de corpos-de- prova cilíndricos. Associação Brasileira de Normas Técnicas - ABNT,NBR 7222.

Bergström, D.; Israelsson, S.; Ohman, M.; Dahlqvist, S.A.; Gref, R.; Boman, C. \&Wasterlund, I. (2008) - Effects of raw material particle size distribution on the characteristics of Scots pine sawdust fuel pellets. Fuel Processing Technology, vol. 89, n. 12, p. 1324-1329. http://doi.org/10.1016/j.fuproc.2008.06.001

Brand, A.M. (2010) - Energia de biomassa florestal. 1. a ed. Interciência, Rio de Janeiro. 131 p.

Brasil (2016) - Boletim Mensal de Monitoramento do Sistema Elétrico Brasileiro. Ministério de Minas e Energia: Secretaria de Energia Elétrica Departamento de Monitoramento do Sistema Elétrico, Brasília, 35 p. 
Brito, J.O. \& Barrichello, L.E.G. (1982) - Aspectos técnicos da utilização da madeira e carvão vegetal como combustíveis. In: Seminário de abastecimento energético industrial com recursos florestais. Secretaria da Indústria, Comércio, Ciência e Tecnologia, São Paulo, Brasil, p. 101-137.

Carroll, J.P. \& Finnan J. (2012) - Physical and chemical properties of pellets from energy crops and cereal straws. Biosystems Engineering, vol. 112, n. 2, p. 151-159. http://doi.org/10.1016/j.biosystemseng.2012.03.012

Carvalho, A.M.M.L.; Pereira, B.L.C. \& Souza, M.M. (2013) - Produção de pellets da madeira. In: Santos, F. ; Colodette, J. \& Queiroz, J. H. (Eds.) - Bioenergia \& Biorrefinaria : cana-de-açúcar e espécies florestais. Universidade Federal de Viçosa, Viçosa, p. 380-400.

Chen, L.J.; Xing, L. \& Hana, L. (2009) - Renewable energy from agro-residues in China: solid biofuels and biomass briquetting technology. Renewable \& Sustainable Energy Reviews, vol. 13, n. 9, p. 2689-2695. http:/l doi.org/10.1016/j.rser.2009.06.025

FAO (2015) - Forestry Production and Trade. Food and Agriculture Organization of the United Nations. http:/l www.fao.org/faostat/en/\#data/FO

Farage, R.M.P.; Rezende, A.A.P.; Silva, C.M; Nunes, W.G.; Carneiro, A.C.O.; Vieira, D.B.; Rodrigues \& C.L.S. (2013) - Avaliação do potencial de aproveitamento energético dos resíduos de Madeira e derivados gerados em fábricas do polo moveleiro de UBÁ-MG. Ciência Florestal, vol. 23, n. 1, p. 203-212.

Furtado, T.S.; Valin, M.; Brand, M.A. \& Bellote, A.F.J. (2010) - Variáveis do processo de briquetagem e qualidade de briquetes de biomassa florestal. Pesquisa Florestal Brasileira, vol. 30, n. 62, p. 101-106. http://dx.doi. org/10.4336/2010.pfb.30.62.101

Gonçalves, B.F.; Yamaji, F.M.; Fernandez, B.O.; Da Róz, A.L. \& Floriano, F.S. (2013) - Caracterização e comparação entre diferentes granulometrias de serragem de Eucalyptus grandis para confecção de briquetes. Revista Instituto Florestal, vol. 25, n. 2, p. 205-213.

Konishi, P. A; Yamaji, F.M.; Silva, D.A. \& Wanderley, C.W.C. (2011) - Influência do teor de umidade na confecção de briquetes de serragem de Pinus sp. In: Congresso de Iniciação Cientifica, Universidade Federal de São Carlos, USFSCAR, São Carlos, Brasil. 1 p.

Lehtikangas, P. (2001) - Quality properties of pelletised sawdust, logging residues and bark. Biomass Bioenergy, vol. 20, n. 5, p. 351-360. http://doi.org/10.1016/S0961-9534(00)00092-1

Li, Y. \& Liu, H. (2000) - High-pressure densification of wood residues to form an upgraded fuel. Biomass and Bioenergy, vol. 19, n. 3, p. 177-186. http://doi.org/10.1016/50961-9534(00)00026-X

Liu, Z.J.; Fei, B.H.; Jiang, Z.H.; Cai, Z.Y \&Liu, X. E. (2014) - Important properties of bamboo pellets to be used as commercial solid fuel in China. Wood Science Technology, vol. 48, n. 5, p. 903-917. http://dx.doi.org/10.1007/ s00226-014-0648-x

Protásio, T.P.; Trugilho, P.F.; Siqueira, H.F.; Alves, I.C.N.; Andrade, C.R. \& Guimarães Júnior, J.B. (2015) Caracterização energética de pellets in natura e torrificados produzidos com madeira residual de Pinus. Brazilian Journal of Forestry Research, vol. 35, n. 84, p. 435-442.

Protásio, T.P; Alves, I.C,N; Trugilho, P.F.; Silva, V.O. \& Baliza, A.E.R. (2011) - Compactação de biomassa vegetal visando à produção de biocombustíveis sólidos. Pesquisa Florestal Brasileira, vol. 31, n. 68, p. 273-283. http://dx.doi.org/10.4336/2011.pfb.31.68.273

Quirino, W.F.; Pinha, I.V.O.; Moreira, A.C.O.M.; Souza, F. \& Tomazello Filho, M. (2012) - Densitometria de raios $X$ na análise da qualidade de briquetes de resíduos de madeira. Scientia Forestalis, vol. 40, n. 96, p. 525-536.

Rezende, A. A. P.; Silva, C. M.; Nunes, W. G.; Carneiro, A. C. O.; Vieira, D. B. \& Rodrigues, C. L. S. (2013) - Avaliação do potencial de aproveitamento energético dos resíduos de madeira e derivados gerados em fábricas do polo moveleiro de Ubá - MG. Ciência Florestal, vol. 23, n. 1, p. 203-212. http://dx.doi. org $/ 10.5902 / 198050988454$

Santos, F.; Colodette, J. \& Queiroz, J.H. (2013) - Bioenergia \& Biorrefinaria: cana-de-açúcar \& espécies florestais. 1. a ed. Produção Independente, Viçosa. 551 p.

Santos, R.C.; Carneiro, A.C.O.; Castro, A.F.M.; Castro, R.V.O.; Bianche, J.J.; Souza, M. M. \& Cardoso, M.T. (2011) - Correlações entre os parâmetros de qualidade da madeira e do carvão vegetal de clones de eucalipto. Scientia Forestalis, vol. 39, n. 90, p. 221-230.

Silva, D.A.; Yamaji, F.M.; Barros, J.L.; Da Róz, A.L. \& Nakashima, G.T. (2015) - Caracterização de biomassas para a briquetagem. Revista Floresta, vol. 45, n. 4, p. 713-722. 
Souza, M.M.; Silva, D.A.; Rochadelli, R. \& Santos, R.C. (2012) - Estimativa de poder calorífico e caracterizaçãopara uso energético de resíduos da colheita e do processamento de Pinus taeda. Revista Floresta, vol. 42, n. 2, p. 325-334.

Spanhol, A.; Nones, D.L.; Kumabe, F.J.B. \& Brand, M.A. (2015) - Qualidade dos pellets de biomassa florestal produzidos em Santa Catarina para a geração de energia. Revista Floresta, vol. 45, p. 833-844.

Toscano, G.; Riva, G.; Foppa Pedretti, E.; Corinaldesi, F.; Mengarelli, C. \& Duca, D. (2013) - Investigation on wood pellet quality and relationship between ash content and the most important chemical elements. Journal Biomass and Bioenergy, vol. 56, p. 317-322. http://doi.org/10.1016/j.biombioe.2013.05.012

Vilas Boas, M.A. (2011) - Efeito do tratamento térmico da madeira para produção de briquetes. Dissertação de Mestrado. Universidade Federal de Viçosa, Viçosa. 65 p.

Vinterbäck, J. (2004) - Pellets 2002: the first world conference on pellets. Biomass and Bioenergy, vol. 27, n. 6, p. 513-520. http://doi.org/10.1016/j.biombioe.2004.05.005exe?family=rubiaceae\&scalefamily=Pseudococcidae\&genus $=$ coffea\&scalegenus $=\&$ species $=$ 